\title{
CHOICE OF APPROPRIATE SPACE-TIME CODING SCHEME FOR MIMO SYSTEMS EMPLOYING CHANNEL CODING UNDER BICM
}

\author{
Mohammad-Ali Khalighi ${ }^{1}$, Jean-François Hélard ${ }^{2}$, Salah Bourennane ${ }^{1}$ \\ ${ }^{1}$ Institut Fresnel, UMR CNRS 6133, Marseille, France \\ ${ }^{2}$ Institut d'Électronique et de Télécommunications de Rennes, UMR CNRS 6164, Rennes, France \\ Email: Ali.Khalighi@fresnel.fr, Jean-Francois.Helard@insa-rennes.fr, Salah.Bourennane@fresnel.fr
}

\begin{abstract}
We consider the use of MIMO structures in the fourth generation of cellular networks. We contrast the performances of the two general categories of orthogonal and non-orthogonal space-time schemes while considering a relatively simple iterative detector for the latter case. We show that with perfect channel knowledge, a substantial gain is obtained by using appropriate non-orthogonal schemes, which justifies the increased receiver complexity. The gain is more considerable for a larger number of transmit antennas. This conclusion remains true when channel estimation at receiver is not perfect.
\end{abstract}

\section{INTRODUCTION}

Use of multiple-input multiple-output (MIMO) systems can potentially satisfy the need to high data rate and high quality of service in cellular mobile radio systems. This is, for instance, studied in the European IST-4MORE project [1] that considers the fourth generation of cellular networks. The choice of the appropriate space-time (ST) scheme in signal transmission plays a major role in the system performance.

\subsection{Problem statement}

Many ST schemes have been presented in the literature since a few years (see for instance [2] and the references therein). In most cases, the design and the optimization of the ST schemes have been done in the absence of channel coding. In a practical system, however, channel coding is usually performed in order to increase the robustness against noise and interference (and in part against fading). Now, actually, the promised gain of one ST scheme over another may be too optimistic when we take into account channel coding.

Concerning ST schemes, two main families are orthogonal block codes (OSTBC) [3, 4] and non-orthogonal schemes. Among the numerous already-proposed non-orthogonal ST schemes, we may state the purely spatial multiplexing or VBLAST scheme [5], the linear dispersion (LD) codes [6], and the non-vanishing codes [7]. The interest of OSTBCs is that they can be decoded using a simple optimal detector. However, they suffer from low rate, especially for increased number of transmit antennas.
In practice, to attain a desired spectral efficiency, i.e., data transmission rate, we should adopt the most appropriate scheme by fixing the degrees of freedom of the system, that is, signal constellation, channel coding rate, and ST coding scheme. The answer to the question "what is the most suitable combination" is not obvious for moderate to high spectral efficiencies. In effect, if a low spectral efficiency is required, an OSTBC scheme together with a powerful turbo-code would be a suitable solution, as the reduction in the overall coding rate is best invested in turbo channel codes [8]. To attain high spectral efficiencies with OSTBC schemes, however, we have to use large signal constellations and to reduce the channel coding rate. Use of larger signal constellations complicates the tasks of synchronization and detection at receiver and also results in a higher SNR required to provide a desired bit-errorrate. Higher ST coding rates are offered by non-orthogonal schemes, hence, relaxing the conditions on signal constellation and channel coding. The disadvantage is that the optimal decoding is much more computationally complex. One good solution would be to use a simple (sub-optimal) iterative detector for this purpose. In this way, we may approach the optimal detection performance after few iterations. Nevertheless, the detector remains more complex, as compared to OSTBC case. We should hence investigate if this increased receiver complexity is justified. In other words, we want to see whether or not by using such a detector, we gain in performance with respect to OSTBC choice, and if this gain is considerable enough to convince us to privilege the nonorthogonal solution.

In a first step, we assume perfect channel knowledge at receiver. Then, in a next step, we extend this study to the case of non-perfect channel knowledge at receiver. Actually, in practice, perfect channel knowledge conditions could never been met and we should make sure whether or not the preference of a ST scheme over another is still preserved, taking into account the channel estimation errors.

\subsection{Study framework}

In this study, we consider the downlink transmission with two or four antennas at the base station (BS) and two antennas at the mobile terminal (MT). Notice that the more critical case 


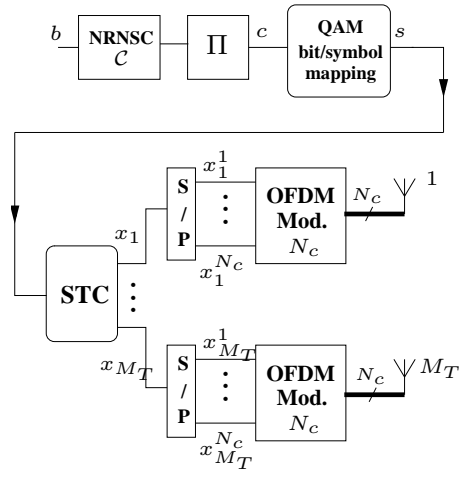

Fig. 1. Block diagram of the transmitter

regarding the computational complexity is in the downlink where constraints on handset power consumption and cost should be satisfied at the MT. At transmitter, channel coding is performed based on bit-interleaved coded modulation (BICM). At receiver, to decode non-orthogonal ST schemes, we use a relatively simple iterative detector based on parallel interference cancellation (PIC). We assume the absence of multi-user interference and use the technique of orthogonal frequency division multiplexing (OFDM) in order to simplify the channel equalization at receiver.

Notice that we are not going to present an exhaustive comparison of all already-proposed non-orthogonal schemes, as this is not the aim of this paper. The question is to choose between the suitable orthogonal and the suitable non-orthogonal schemes. The ST schemes that we consider, are presented and described in Subsection 3.1 where we also explain the reason that we consider them in our study. We will see that for some of the considered non-orthogonal schemes, the iterative detector does not converge properly.

The paper is organized as follows. Section 2 presents our system model. In Section 3 we present different ST schemes that we consider in this work, and also describe the data detection at receiver. Simulation results are presented in Section 4 to compare the performance obtained by different ST schemes for several spectral efficiencies. Two cases of perfect channel estimation and pilot-only-based channel estimation are considered. Section 5 concludes the paper.

\section{SYSTEM MODEL}

We denote by $M_{T}$ and $M_{R}$ the number of antennas at transmitter and at receiver, respectively. We have $M_{R}=2$ and $M_{T}=2$ or $M_{T}=4$. Fig. 1 shows the block diagram of the transmitter. A non-recursive non systematic convolutional (NRNSC) code, random interleaving, and Gray-coded QAM modulation with $B$ bits per symbol are considered. Power normalized symbols $s$ are combined according to a given ST scheme. The resulting symbols are then passed through the OFDM modulator of $N_{c}$ sub-carriers before transmission. We assume frequency non-selective fading per sub-carrier and the absence of inter-symbol interference and inter-carrier inter- ference. MIMO channel fading coefficients corresponding to each sub-carrier are assumed to be independent and Rayleigh distributed. Let $\boldsymbol{S}$ of dimension $(Q \times 1)$ be the vector of data symbols prior to $\mathrm{ST}$ coding, $\boldsymbol{S}=\left[s_{1}, s_{2}, \cdots, s_{Q}\right]^{t}$, where ${ }^{t}$ stands for vector or matrix transpose. By ST coding, $\boldsymbol{S}$ is mapped into a matrix $\boldsymbol{X}$ of dimension $\left(M_{T} \times T\right), T$ being the number of channel uses. The ST coding rate is defined as $R_{S T C}=Q / T$. A frame of $N$ encoded bits, $N$ being the interleaver size, corresponds to $\frac{N T}{B Q}$ channel uses after ST coding. We define $N_{F}$, the number of OFDM symbols per frame, $N_{F}=N T /\left(B Q N_{c}\right)$. We assume perfect interleaving before OFDM modulation. Channel coefficients, independent for each sub-carrier, are assumed to be constant during $N_{F}$ OFDM symbols, and to change to new independent values from one frame to next.

\section{SPACE-TIME CODING AND DECODING}

\subsection{Considered ST schemes}

For $M_{T}=2$, as the most suitable orthogonal scheme, we consider the Alamouti code [3] with $Q=M_{T}=T=2$ and $R_{S T C}=1$, given below.

$$
\boldsymbol{X}=\left[\begin{array}{rr}
s_{1} & s_{2} \\
-s_{2}^{*} & s_{1}^{*}
\end{array}\right]
$$

As the non-orthogonal scheme, we consider the simplest scheme, i.e., V-BLAST, for which, $Q=2, T=1$ and $R_{S T C}=2$ : $\boldsymbol{X}=\left[\begin{array}{ll}s_{1} & s_{2}\end{array}\right]^{t}$. We also consider the optimized golden code (denoted here by GLD), presented in [7], which offers fullrate full-diversity and has the property of non-vanishing determinant. For this code, described below, we have $Q=4$, $T=2$, and $R_{S T C}=2$.

$$
\boldsymbol{X}=\frac{1}{\sqrt{5}}\left[\begin{array}{rr}
\alpha\left(s_{1}+\theta s_{2}\right) & \alpha\left(s_{3}+\theta s_{4}\right) \\
\gamma \bar{\alpha}\left(s_{3}+\bar{\theta} s_{4}\right) & \bar{\alpha}\left(s_{1}+\bar{\theta} s_{2}\right)
\end{array}\right]
$$

where $\theta=\frac{1+\sqrt{5}}{2}, \alpha=1+j(1-\theta), \bar{\theta}=1-\theta, \bar{\alpha}=$ $1+j(1-\bar{\theta}), \gamma=j, j=\sqrt{-1}$. The factor $1 / \sqrt{5}$ in (2) ensures normalized transmit power per channel use.

For $M_{T}=4$, as the orthogonal choice, we perform Alamouti coding alternatively on one pair of antennas, while turning the other pair off. This scheme is called time-switched Alamouti and denoted here by $\mathrm{Sw}-\mathrm{Al}$. For the $\mathrm{Sw}-\mathrm{Al}$ scheme, given by (3), we have $Q=M_{T}=T=4$ and $\boldsymbol{R}_{S T C}=1$. Notice that we would prefer this code to that proposed in [4], equation (38), since the rate of that code is $1 / 2$.

$$
\boldsymbol{X}=\sqrt{2}\left[\begin{array}{rrrr}
s_{1} & s_{2} & 0 & 0 \\
-s_{2}^{*} & s_{1}^{*} & 0 & 0 \\
0 & 0 & s_{3} & s_{4} \\
0 & 0 & -s_{4}^{*} & s_{3}^{*}
\end{array}\right]
$$

The first non-orthogonal scheme that we consider is the simple double-Alamouti code, denoted here by $\mathrm{D}-\mathrm{Al}$ and given in (4). For D-Al code, we have $Q=4, T=2$, and $R_{S T C}=2$. 


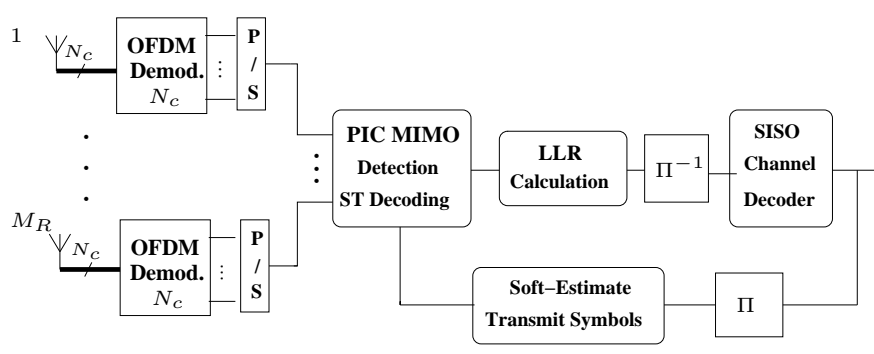

Fig. 2. Block diagram of the receiver

$$
\boldsymbol{X}=\left[\begin{array}{rrrr}
s_{1} & -s_{2}^{*} & s_{3} & -s_{4}^{*} \\
s_{2} & s_{1}^{*} & s_{4} & s_{3}^{*}
\end{array}\right]^{t}
$$

We also consider the LD code proposed in [6], optimized for $M_{T}=4$ and $M_{R}=2$ by maximizing the mutual information between transmitted and received signals. For this code, denoted here by $\mathrm{LD}_{4 \times 2}$, we have $Q=12, T=6$ and $R_{S T C}=2$. Its generator matrix is not presented here due to space limit. Note that the simple V-BLAST scheme cannot be used for $M_{T}=4$ since $M_{R}=2<M_{T}$.

\subsection{Decoding of ST codes}

The block diagram of the receiver is shown in Fig.2. With the assumptions made in Section 2, we can ignore the ensemble of the operations of demultiplexing, OFDM modulation and demodulation, and multiplexing. Transposing hence the frequency dimension into the time dimension, the MIMO OFDM channel can equivalently be considered as a singlecarrier block fading MIMO channel. In this way, we can describe our channel by a matrix $\boldsymbol{H}$ of dimension $\left(M_{R} \times M_{T}\right)$, invariable over a block of $N_{F}$ channel uses. A frame of $N$ bits corresponds hence to $N_{c}$ blocks with independent fades, each block corresponding to a given sub-carrier in the original OFDM model. Now, corresponding to a ST encoded matrix $\boldsymbol{X}$, we receive the matrix $\boldsymbol{Y}$ of dimension $\left(M_{R} \times T\right)$.

\subsubsection{Formulation of data transmission}

We separate the $\Re$ and $\Im$ parts of the entries of $\boldsymbol{S}, \boldsymbol{X}$, and $\boldsymbol{Y}$ and stack them row-wise in vectors $\mathcal{S}$ of dimension $(2 Q \times 1)$, $\mathcal{X}$ of dimension $\left(2 M_{T} T \times 1\right)$, and $\mathcal{Y}$ of dimension $\left(2 M_{R} T \times\right.$ $1)$, respectively. For instance,

$$
\mathcal{S}=\left[\Re\left\{s_{1}\right\} \Im\left\{s_{1}\right\}, \cdots, \Re\left\{s_{Q}\right\} \Im\left\{s_{Q}\right\}\right]^{t} .
$$

We obtain hence $\mathcal{X}=\mathcal{F} \mathcal{S}$, where the matrix $\mathcal{F}$ of dimension $\left(2 M_{T} T \times 2 Q\right)$ depends on the ST scheme (see [6]). On the other hand, we can write $\mathcal{Y}=\mathcal{H} \mathcal{X}+\mathcal{N}$, where $\mathcal{N}$ is the vector of real AWGN of zero mean and variance $N_{0}$, and the matrix $\mathcal{H}$ of dimension $\left(2 M_{R} T \times 2 M_{T} T\right)$ is constructed from the $\Re$ and $\Im$ parts of the entries $h_{i j}$ of the initial matrix $\boldsymbol{H}$ (see [6] for details). We can consider an equivalent channel matrix $\mathcal{H}_{e q}$ of dimension $\left(2 M_{R} T \times 2 Q\right)$ such that:

$$
\mathcal{Y}=\mathcal{H} \mathcal{F} \mathcal{S}+\mathcal{N}=\mathcal{H}_{e q} \mathcal{S}+\mathcal{N}
$$

\subsubsection{MIMO detection: ST decoding}

The detection problem is to find the transmitted data vector $\mathcal{S}$, given the vector $\mathcal{Y}$. The iterative detector is composed of four main blocks, i.e., PIC detector, LLR calculation, softinput soft-output (SISO) channel decoder, and transmit symbols soft estimation, described in [9, 10] (see Fig.2). SISO decoding is based on the Max-Log-MAP algorithm. Concerning the PIC detector, at the first iteration, the detected symbols $\hat{s}_{q}$ are obtained via MMSE filtering [10]:

$$
\hat{s}_{q}=h_{q}^{t}\left(\mathcal{H}_{e q} \mathcal{H}_{e q}^{t}+\sigma_{n}^{2} \boldsymbol{I}\right)^{-1} \mathcal{Y}
$$

where $h_{q}$ of dimension $\left(2 M_{R} T \times 1\right)$ is the $q^{t h}$ column of $\mathcal{H}_{e q}$. From the second iteration, we calculate soft estimates of the transmit symbols $\tilde{\mathcal{S}}$ using decoder soft outputs and perform interference cancelling followed by zero-forcing detection:

$$
\hat{\mathcal{Y}}_{q}=\mathcal{Y}-\mathcal{H}_{q} \tilde{\mathcal{S}}_{q} \quad \rightarrow \quad \hat{s}_{q}=\frac{1}{h_{q}^{t} h_{q}} h_{q}^{t} \hat{\mathcal{Y}}_{q}
$$

where $\tilde{\mathcal{S}}_{q}$ of dimension $((2 Q-1) \times 1)$ is $\tilde{\mathcal{S}}$ with its $q^{\text {th }}$ entry removed, and $\mathcal{H}_{q}$ of dimension $\left(2 M_{R} T \times(2 Q-1)\right)$ is $\mathcal{H}_{e q}$ with its $q^{\text {th }}$ column removed. For the case of orthogonal ST schemes the decoding is performed using (7) only.

For logarithmic likelihood ratio (LLR) calculation, we assume Gaussian noise plus residual interference (RI) after PIC detection [9]. Note that, as the detection is performed on blocks of $Q$ complex symbols, or in other words on blocks of $2 Q$ real symbols in our model, the RI comes in fact from $(2 Q-1)$ other real symbols in the corresponding block. This is, of course, the case only for non-orthogonal ST coding. Now, in LLR calculation, we need the variance of noise plus RI [9]. This variance is calculated for the first iteration. For next iterations, however, we cannot calculate it analytically. To take into account the RI, we should hence estimate the corresponding variance in each iteration and for each one of $2 Q$ real symbols [11]. Here, to simplify the detector further, we do not estimate this variance, and we consider only the noise variance except for the first iteration. In other words, we suppose perfect interference cancellation in succeeding iterations. We will later see that this simplification affects considerably the performance of certain ST schemes.

\section{SIMULATION RESULTS}

For different ST schemes we compare curves of bit-errorrate (BER) versus $E_{b} / N_{0}$ for a given spectral efficiency $\eta$ (in bps/Hz). $E_{b} / N_{0}$ includes the receiver array gain. To have the same $\eta$ for different ST schemes, we accordingly set the signal constellation and the channel coding rate $R_{c}$. NRNSC code $(133,177)_{8}$ is considered where without puncturing, $R_{c}=1 / 2$. Different $\mathrm{ST}$ schemes we consider are resumed in Table I. We set $N_{c}$ to 32 , which is the frequency diversity order with the assumptions made in Section 2, and set $N_{F}$ to 24 . 
Table 1. Different MIMO and ST schemes

\begin{tabular}{|c|c|c|c|c|}
\hline \multirow{2}{*}{$\begin{array}{c}(2 \times 2) \\
\eta=2\end{array}$} & Alamouti & 1 & $16-$ QAM & $1 / 2$ \\
\cline { 2 - 5 } & V-BLAST & 2 & QPSK & $1 / 2$ \\
\cline { 2 - 5 } & GLD & 2 & QPSK & $1 / 2$ \\
\hline \hline \multirow{2}{*}{$\begin{array}{c}(2 \times 2) \\
\eta=3\end{array}$} & Alamouti & 1 & $16-$ QAM & $3 / 4$ \\
\cline { 2 - 5 } & V-BLAST & 2 & QPSK & $3 / 4$ \\
\cline { 2 - 5 } & GLD & 2 & QPSK & $3 / 4$ \\
\hline \hline \multirow{2}{*}{$\begin{array}{c}(4 \times 2) \\
\eta=4\end{array}$} & Sw-Al & 1 & $64-$ QAM & $2 / 3$ \\
\cline { 2 - 5 } & D-Al & 2 & $16-$ QAM & $1 / 2$ \\
\cline { 2 - 5 } & LD $_{4 \times 2}$ & 2 & $16-$ QAM & $1 / 2$ \\
\hline
\end{tabular}

\subsection{Perfect channel knowledge at receiver}

For $M_{T}=2$ and two cases of $\eta=2$ and $\eta=3$, performance curves are shown in Figures 3 and 4, respectively. For BLAST and GLD schemes we have shown BER curves after two iterations and after the full convergence of the detector, i.e., four iterations. We see that for $\eta=2$, by using BLAST scheme, we gain about $3.3 \mathrm{~dB}$ and $3.75 \mathrm{~dB}$ in $\mathrm{SNR}$ at $\mathrm{BER}=10^{-4}$ after two and four iterations, respectively, compared to Alamouti coding. The corresponding gains by using GLD code are about $3.5 \mathrm{~dB}$ and $4.3 \mathrm{~dB}$, respectively. We note also that even when for the reasons of complexity and/or latency, only two iterations are to be performed, the gain in SNR compared to Alamouti scheme is still considerable.

For $\eta=3$, we see that the BLAST scheme undergoes an important performance degradation, as compared to GLD code. We verified that this is due to the fact that we neglected RI for iterations more than one in LLR calculation (see Subsection 3.2.2 and [11]). The present case is more critical than the case of $\eta=2$, because with $R_{c}=3 / 4$ we do not perform "enough" coding. Interestingly, for the full-diversity GLD code, however, the detector converges properly. This can be justified by a better interference rejection by GLD code as it offers more diversity gain. We verified this by analyzing the histograms of noise plus RI at the PIC detector output. These histograms are close to Gaussian for GLD code but this is not the case for BLAST scheme. The gains obtained by using GLD compared to Alamouti scheme are about $2.06 \mathrm{~dB}$ and $3.7 \mathrm{~dB}$ after two and four iterations, respectively.

For $M_{T}=4$ and $\eta=4$, performance curves are shown in Fig.5. Concerning D-Al and $\mathrm{LD}_{4 \times 2}$ schemes, about four iterations are necessary to attain the full detector convergence. We notice that $\mathrm{D}-\mathrm{Al}$ has a better performance, compared to $\mathrm{LD}_{4 \times 2}$ code. We again verified that this is due to sub-optimal LLR calculation. For $\mathrm{LD}_{4 \times 2}$ code, $Q$ is much larger than that for $\mathrm{D}-\mathrm{Al}$, and hence, the interference is more important. Another disadvantage of $\mathrm{LD}_{4 \times 2}$ scheme is that, $T$ is much larger for this code, and consequently, the detector becomes more computationally complex at the first iteration (notice the need of matrix inversion in (7)). The gain achieved by D-Al compared to $\mathrm{Sw}-\mathrm{Al}$ scheme is about $5.55 \mathrm{~dB}$ and $6.7 \mathrm{~dB}$, after two and four iterations, respectively.

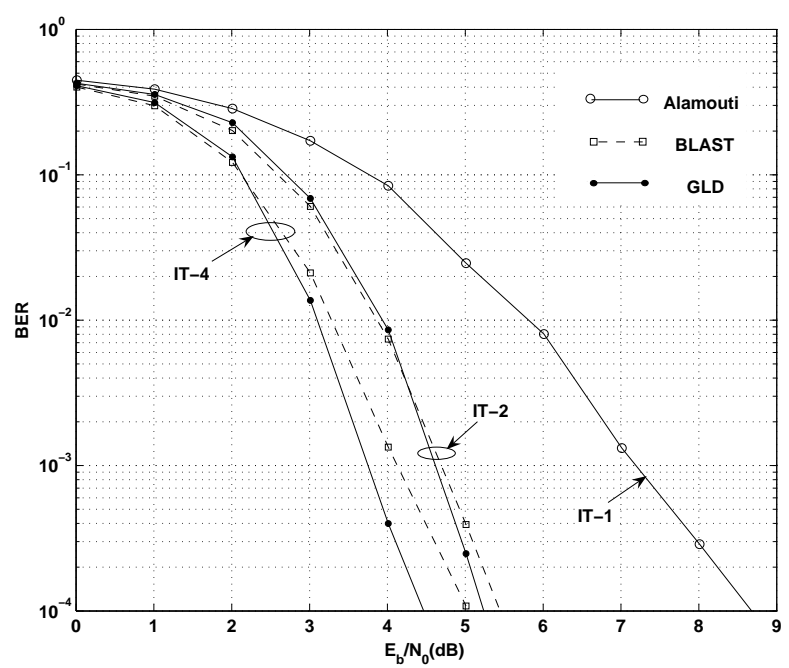

Fig. 3. Performance comparison, $M_{T}=M_{R}=2, \eta=2 \mathrm{bps} / \mathrm{Hz}$

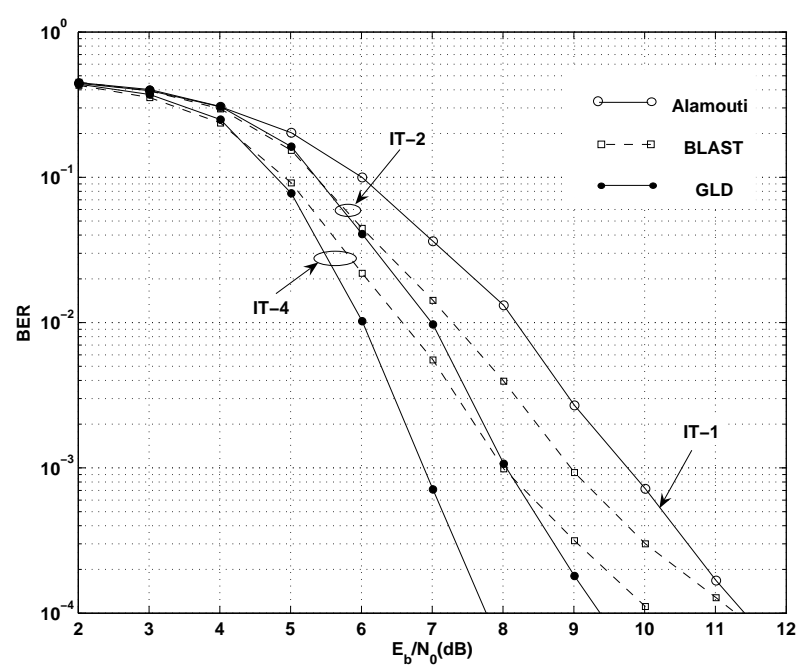

Fig. 4. Performance comparison, $M_{T}=M_{R}=2, \eta=3 \mathrm{bps} / \mathrm{Hz}$

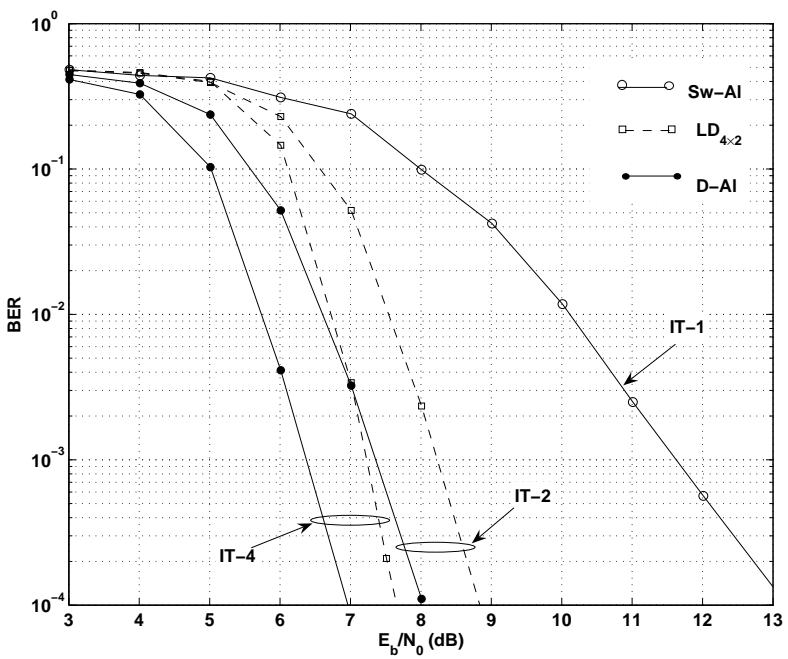

Fig. 5. Performance comparison, $M_{T}=4, M_{R}=2, \eta=4 \mathrm{bps} / \mathrm{Hz}$. 


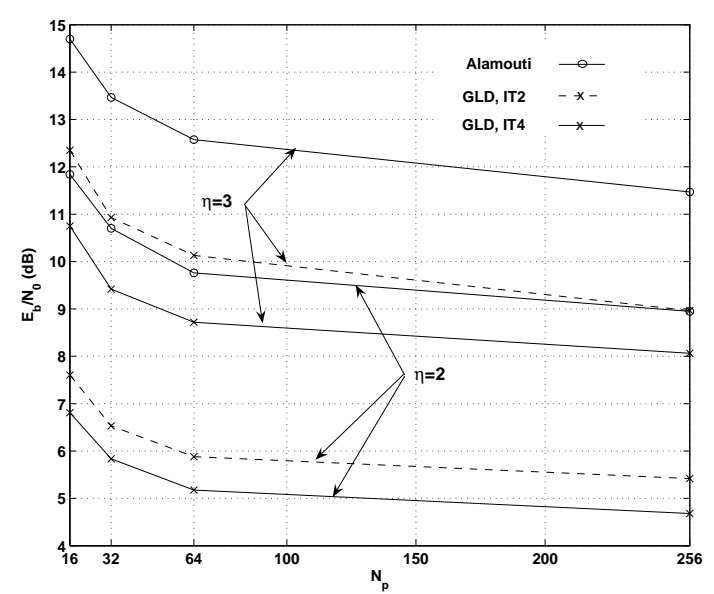

Fig. 6. SNR required to attain $\mathrm{BER}=10^{-4}, M_{T}=M_{R}=2$, same conditions as in Fig.3.

\subsection{Pilot-only-based channel estimation}

It is important to study the effect of channel estimation errors on the above-presented results. Actually, lower-rate orthogonal schemes would be more sensitive to channel estimation errors as they have to use a larger constellation set to attain a desired spectral efficiency. Non-orthogonal schemes may in turn be more sensitive to these errors, as they affect the iterative detector convergence.

Here, we consider pilot-only-based channel estimation. Using $N_{p}$ pilot bits, we devote $N_{p} /\left(B M_{T}\right)$ channel uses to the transmission of optimal mutually orthogonal QPSK pilot sequences from $M_{T}$ transmit antennas [9]. Like data symbols, pilot symbols are also normalized in power. We assume that $N_{0}$ is known at receiver and pilots are used only for the estimation of $\boldsymbol{H}$. Figures 6 and 7 show the SNR required to attain the BER of $10^{-4}$ for the cases of $M_{T}=2$ and $M_{T}=4$, respectively, considering the second iteration and the full convergence of the detector. Only GLD and D-Al are considered as appropriate non-orthogonal schemes. Note that $\eta$ does not take into account the pilots. Also, note that we take $N_{p}$ a little greater than the required value for channel identifiability (see [9] for details). We notice that the gain obtained by using non-orthogonal w.r.t. orthogonal schemes is still considerable and even more important for relatively small $N_{p}$ values.

\section{CONCLUSIONS}

We considered the performance of orthogonal and non-orthogonal ST schemes when used with a convolutional channel code under BICM. We showed that in both cases of perfect channel knowledge and estimated channel at receiver, a substantial gain can be obtained compared to orthogonal coding, by using appropriate non-orthogonal ST schemes and a simple iterative detector. This is true even if few iterations are to be processed in order to keep the system complexity and latency reasonable.

\section{ACKNOWLEDGMENT}

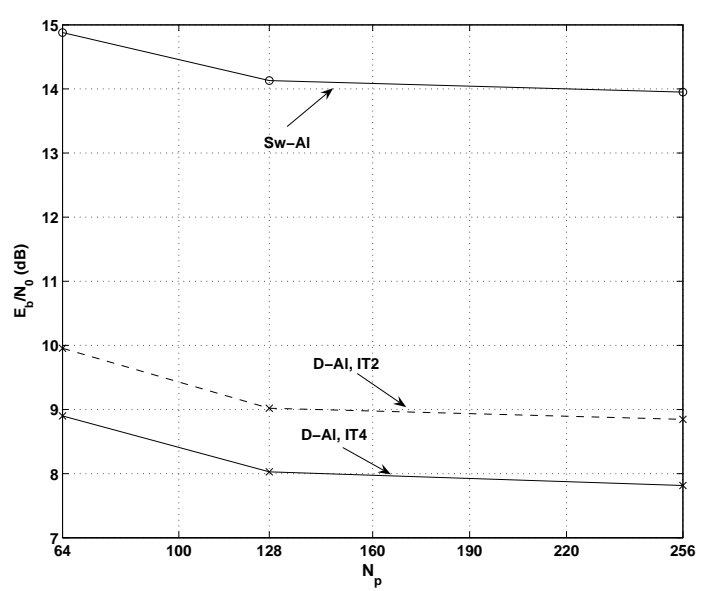

Fig. 7. SNR required to attain $\mathrm{BER}=10^{-4}, M_{T}=4, M_{R}=2$, same conditions as in Fig.5.

This work was partly supported by the European IST-FP6-4MORE project (4G MC-CDMA Multiple Antenna System On Chip for Radio Enhancements).

\section{REFERENCES}

[1] "IST 4MORE project (4G MC-CDMA Multiple Antenna System On Chip for Radio Enhancements)," http://www.ist4more.org .

[2] B. Vucetic and J. Yuan, Space-Time Coding, John Wiley \& Sons Ltd, Chichester, England, 2003.

[3] S.M. Alamouti, "A simple transmit diversity technique for wireless communications," IEEE J. Sel. Areas Commun., vol. 16, no. 8, pp. 1451-1458, Oct. 1998.

[4] V. Tarokh, H. Jafarkhani, and A.R. Calderbank, "Space-time block codes from orthogonal designs," IEEE Trans. Info. Th., vol. 45, no. 5, pp. 1456-1467, July 1999.

[5] G.D. Golden, G.J. Foschini, R.A. Valenzuela, and P.W. Wolniansky, "Detection algorithm and initial laboratory results using V-BLAST space-time communication architecture," Elect. Lett., vol. 35, no. 1, pp. 14-16, Jan. 1999.

[6] B. Hassibi and B.M. Hochwald, "High-rate codes that are linear in space and time," IEEE Trans. Info. Th., vol. 48, no. 7, pp. 1804-1824, July 2002.

[7] J.-C. Belfiore, G. Rekaya, and E. Viterbo, "The golden code: a $2 \times 2$ full-rate space-time code with nonvanishing determinants," IEEE Trans. Info. Th., vol. 51, no. 4, pp. 1432-1436, Apr. 2005.

[8] V. Le Nir, J-M. Auffray, M. Hélard, J-F. Hélard, and R. Le Gouable, "Combination of space-time block coding with MCCDMA technique for MIMO systems with two, three and four transmit antennas," IST Mobile Commun. Summit, June 2003.

[9] M.A. Khalighi and J. Boutros, "Channel estimation in turboBLAST detectors using EM algorithm," ICASSP Conf., vol. III, pp. 1037-1040, Mar. 2005, Philadelphia, PA.

[10] M. Sellathurai and S. Haykin, "Turbo-BLAST for wireless communications: theory and experiments," IEEE Trans. Sig. Proc., vol. 50, no. 10, pp. 2538-2546, Oct. 2002.

[11] M.A. Khalighi and J.-F. Hélard, "Should MIMO orthogonal space-time coding be preferred to non-orthogonal coding with iterative detection?," ISSPIT Conf., pp. 340-345, Dec. 2005. 